\title{
Storm forecasting: additional lessons from the CD28 superagonist TGN 1412 trial
}

Christopher Horvath, Laura Andrews, Andreas Baumann, Lauren Black, Diann Blanset, Joy Cavagnaro, Kenneth L. Hastings, David L. Hutto, Timothy K. MacLachlan, Mark Milton, Theresa Reynolds, Stan Roberts, Mark Rogge, Jennifer Sims, George Treacy, Garvin Warner and James D. Green

This letter is in response to the Comment article published in Nature Reviews Immunology by Thomas Hünig (The storm has cleared: lessons from the CD28 superagonist TGN1412 trial. Nature Rev. Immunol. 12, 317-318 (2012)) $)^{1}$. In his commentary, Thomas Hünig attempts to explain "why the three sets of preclinical data ... used to support this Phase I clinical trial had failed to predict the cytokine storm" in healthy volunteers. He describes some of the findings in these test systems and concludes that they "all failed to provide evidence for the toxic potential of the antibody for distinct and unrelated reasons". He further claims that "the disastrous outcome ... may have made a positive contribution to the future development of immunomodulatory drugs", primarily by "teaching us the superiority of the MABEL [minimum anticipated biological effect level]-based approach over the NOAEL [no observed adverse effect level]-based approach".

In sharp contrast to Thomas Hünig's opinions, we believe that the serious adverse events were predictable based on the known CD28 receptor biology, the intended pharmacological activity of TGN1412 and the preclinical safety assessment programme. The purpose of a preclinical safety assessment programme is to identify potential safety risks and to qualify and quantify these risks by using in vitro human data, in vitro and in vivo animal data, and translational and mechanism-based knowledge of the intended pathway modulation to 'predict' in vivo human responses. We feel that these criteria were not met for TGN1412. Specifically, the publicly available information in the investigational medicinal product dossier (IMPD) and/or the investigator's brochure demonstrates deficiencies in four major areas of the preclinical safety assessment. One, there was a failure to identify risk based on the intended target, mechanism of action and previous experiences with comparator products. Two, there was a failure to reconcile discordant preclinical data sets. Three, there was a failure to identify a pharmacologically relevant species and/or data set for the determination of the first-in-human dose. And, four, there was a failure to translate the preclinical data to estimate the anticipated pharmacological activity of the first-in-human dose. We shall discuss these topics in order.

Failure to identify risk based on the intended target and mechanism of action. With TGN1412, there were strong reasons to proceed with caution based on the nature of the target and the intended and/or desired pharmacological activity: superactivation of T cells. Comparable superactivation of T cells occurs naturally when bacterial superantigens bypass normal antigen presentation processes and directly co-activate MHC class II molecules and T cell receptors (TCRs), resulting in fever, extensive cytokine release, toxic shock and death at plasma concentrations as low as $0.1 \mathrm{pg}$ per ml (REF. 2). Historically, iatrogenic T cell mitogenesis, $T$ cell superactivation and sudden, but prolonged, depletion of circulating $\mathrm{T}$ cells have been the hallmarks of immunomodulatory CD3-specific monoclonal antibodies, including OKT3 (Janssen-Cilag), HuM291 (visilizumab; PDL BioPharma) and others ${ }^{3}$. These reactions depend on the extent of receptor occupancy and can occur at remarkably low doses. Although TGN1412 was a first-in-class CD28-specific superagonist, it was intended to induce the superactivation of $\mathrm{T}$ cells, much like the CD3-specific antibodies that induce cytokine-release syndrome (CRS). While cursory mention of the possibility of CRS was made in the IMPD and investigator's brochure, insufficient consideration was given to the lessons already learnt from natural superagonists and comparator products. In fact, it was stated in the investigator's brochure that "TGN1412 is expected to be well tolerated in humans and not to elicit any adverse effects". Indeed, the design of the Phase I study, in which all the volunteers were dosed at 10-minute intervals ${ }^{4}$, suggests that no practical consideration was given to the possibility of CRS occurring.

Failure to reconcile discordant preclinical data. The early decision by the Medicines and Health Regulatory Agency ${ }^{5}$ to publicly release the IMPD, investigator's brochure, clinical trial protocol, assessment report and informed consent for TGN1412 (available on the Citizens for Responsible Care and Research website) should be applauded because it facilitated independent review. Such a review shows that disparate results were obtained from in vitro and in vivo assessments of the superagonist activity of CD28-specific antibodies in humans, rats and monkeys.

In vitro, $\mathrm{T}$ cell activation and cytokine release have been demonstrated for the monoclonal antibody 5.11A1 (a precursor to TGN1412) in human systems and for JJ316 (a rat CD28-specific monoclonal antibody homologous to TGN1412) in rat systems ${ }^{6,7}$. Based on the publicly released information, it appears that no such demonstration of in vitro pharmacological activity of TGN1412 was presented for human or monkey T cells in the IMPD or investigator's brochure. In these documents, it appears that the only data describing the activity of TGN1412 in human systems were the results for in vitro $\mathrm{T}$ cell mitogenesis assays that were conducted with the precursor monoclonal antibody 5.11A1 or with TGN1412 in the presence or absence of methotrexate. The concentration-response relationships for TGN1412 in human cell systems and their relationship (or lack thereof) to those for TGN1412 in monkey systems or JJ316 in rat systems do not appear to have been discussed, limiting the ability to model potential effects of the administration of TGN1412 to humans from the in vitro data.

In rats, a single dose of $5 \mathrm{mg}$ per $\mathrm{kg}$ of JJ316 resulted in a 3- to 6 -fold increase in spleen size and a 20 -fold increase in splenic regulatory $\mathrm{T}\left(\mathrm{T}_{\mathrm{Reg}}\right)$ cell numbers by day 3 ; circulating $\mathrm{T}$ cells were not evaluated ${ }^{8}$. This was the expected pharmacological outcome for a CD28-specific superagonist antibody in a rat system, and was consistent with the in vitro activity. By contrast, ascending single-dose pharmacokinetic and pharmacodynamic studies with 5 to $50 \mathrm{mg}$ per kg of TGN1412 in monkeys resulted in the saturation of $\mathrm{CD} 28$ receptors on T cells, but caused only minimal $(<3$-fold) and delayed (by $>2$ weeks) increases in 
circulating $\mathrm{T}_{\text {Reg }}$ cell numbers and no doserelated cytokine response. In the pivotal toxicology study in monkeys, repeated weekly doses of $5 \mathrm{mg}$ per $\mathrm{kg}$ and $50 \mathrm{mg}$ per $\mathrm{kg}$ of TGN1412 resulted in saturation of the receptor at both doses. However, TGN1412 was reported to have induced the expansion of T cell populations only at the $5 \mathrm{mg}$ per kg dose, and then only in males $(n=3)$. There was no splenomegaly and splenic $\mathrm{T}_{\text {Reg }}$ cells were not evaluated. Thus, despite complete receptor occupancy, there was no dose-responsive, biologically relevant pharmacological activity and certainly no demonstration of the desired superagonist activity. This was consistent with the lack of in vitro activity against monkey cells that was later demonstrated in 2007 (REF. 9). When considered as a whole, the four data sets in the regulatory filings (in vitro studies in rats and humans and in vivo studies in rats and monkeys) indicate that there was a significant issue with the pharmacological relevance of monkeys.

In addition, there was one other data set that was available to TeGenero before the initiation of the Phase I trial but that was probably not available to regulators, as it was not apparent in the IMPD. A paper that included an author from TeGenero ${ }^{10}$ was submitted for publication on 17 January 2006, just 1 month after the 19 December 2005 signature dates on the IMPD and investigator's brochure documents, and was published online on 2 March 2006, just 11 days before volunteers received doses of $0.1 \mathrm{mg}$ per $\mathrm{kg}$ of TGN1412. In this work, CD28 superagonism was evaluated in mice lacking a functional immune system that had been irradiated and reconstituted with human CD34+ fetal liver stem cells. These 'humanized' mice (termed HIS mice) develop immune systems containing all the major human myeloid and lymphoid cellular compartments, including human $\mathrm{CD} 28^{+} \mathrm{T}$ cells and antigen-presenting cells expressing human $\mathrm{Fc}$ receptors ${ }^{10}$. In these mice, administration of as little as $\sim 0.3 \mathrm{mg}$ per $\mathrm{kg}$ of 5.11A1 resulted, by day 6 , in a 7 -fold increase in the overall number of human thymocytes and a 20-fold increase in the number of mature thymocytes, which was said to be consistent with the intended superagonist effect. However, the mice also showed a "drastic" depletion of circulating human $\mathrm{CD} 28^{+} \mathrm{T}$ cells from between day 3 and day 60 after antibody administration ${ }^{10}$. The authors of this study pointed out that they had not observed such an effect of 5.11A1 previously and they "hypothesized that human $\left[\mathrm{CD} 28^{+}\right] \mathrm{T}$ cells found in the periphery of HIS mice could be particularly sensitive to stimuli". We consider this depletion of circulating human $\mathrm{T}$ cells to be analogous to that observed with numerous CD3-specific monoclonal antibodies and to be strongly suggestive of overwhelming CD28-mediated T cell superactivation. As the authors considered this to be a new finding, a thorough consideration of the potential implications for humans and the submission of a report to the regulatory authorities was warranted before the initiation of the clinical trial.

As the examples from the IMPD and other TeGenero publications illustrate, the different in vitro and in vivo preclinical test systems gave conflicting results that were not reconciled. Superagonism was demonstrated in vivo with JJ316 in rats and with 5.11A1 in HIS mice and in vitro with JJ316 on cells expressing rat CD28 and with 5.11A1 on cells expressing human CD28. However, pharmacological activity was not demonstrated in monkeys, warranting further investigation to reconcile this discrepancy.

\section{Failure to identify a pharmacologically relevant test system (or systems) for} first-in-human dose selection. As indicated above, expected pharmacological effects were seen in rodent systems using precursor or homologous antibodies. However, within the regulatory filings for TGN1412, there were no in vitro pharmacology data comparing the results for human cells with those from monkey cells. Monkeys appeared to have been selected as the relevant species solely on the basis of the demonstration that TGN1412 binds to monkey CD28 (from receptor occupancy and tissue cross-reactivity studies) and the conserved sequence of the CD28 6-aminoacid C"D loop, without any data to indicate that TGN1412 exhibits relevant pharmacological activity in monkeys. As relevant pharmacological effects were not seen, an alternative approach to first-in-human dose selection focusing on the data from other assay systems would have been appropriate.

Failure to estimate the anticipated activity of the first-in-human dose. The nature of the target, the pertinent historical experience with relevant comparator products and the conflicting preclinical data with TGN1412 would indicate a distinct likelihood of CRS. This understanding would dictate that a conservative approach should be taken and that the first-in-human dose for TGN1412 should be soundly justified on the basis of anticipated pharmacological activity.
However, a discussion of the potential pharmacological effect of the first-in-human dose was not apparent in the IMPD. In fact, there appears to be no discussion of the pharmacokinetic or pharmacodynamic effects of any of the proposed Phase I doses $(0.1,0.5,2$ and $5 \mathrm{mg}$ per $\mathrm{kg})$ and no projection of the anticipated human therapeutic dose or concentrations. The first-in-human dose was simply proposed as $0.1 \mathrm{mg}$ per $\mathrm{kg}$, or $1 / 500$ of the NOAEL in monkeys.

Obviously, for successful implementation, a NOAEL approach requires that one is able to 'observe' an adverse effect, which is not possible when pharmacological activity is not present in the chosen preclinical species. Hünig further implies that reliance on a NOAEL approach alone was the accepted practice at the time, and that the subsequent recommendation for and adoption of the MABEL approach was a positive contribution of the TeGenero incident. This perspective is not consistent with the fact that applicable regulatory guidance was in place at the time for biotherapeutics products, for which toxicity typically arises from exaggerated pharmacological effects. This guidance stressed that pharmacologically relevant test systems must be identified and used for safety assessment ${ }^{11}$ and that the NOAELderived human equivalent (first) dose should be revised if a human equivalent dose based on a pharmacologically active dose (PAD; the precursor to MABEL) would be a more sensitive indicator of potential toxicity ${ }^{12}$.

Reasons for additional conservatism when selecting the first-in-human dose are specifically listed in the US Food and Drug Administration (FDA) guidance. These reasons include: a new therapeutic target, a long half-life of the product, inadequate dose-response data and an animal model (species) with limited utility (pharmacological relevance) ${ }^{12}$. Additional guidelines, in place since 1977, stipulate that the first-in-human dose should not exceed the projected human therapeutic dose ${ }^{13}$. Subsequent analyses of the TGN1412 dose, using data available before the trial, showed that the $0.1 \mathrm{mg}$ per kg first-in-human dose was estimated to result in a maximum plasma concentration of $\sim 1 \mu \mathrm{g}$ per $\mathrm{ml}$ and to be associated with near-complete receptor saturation $^{14-16}$. This would be predictably expected to result in overwhelming $\mathrm{T}$ cell superactivation and risk of CRS, as is common to T cell-activating biotherapeutics.

In conclusion, we believe that Hünig's commentary perpetuates the mistaken belief that the TeGenero event was "completely unpredicted by the preclinical studies". We 
believe that serious adverse events, including CRS, were indeed predictable, or should have been anticipated, based on the known CD28 receptor biology and the intended pharmacological activity of TGN1412. Failure to reconcile inconsistencies in the available data and to correctly apply a translational approach using the most relevant data for selection of the first-in-human dose compounded this problem. Our perspective is that the evolution of regulatory guidance and industry practice is best facilitated by scientific discussions that include parties experienced in pharmaceutical development and regulation, not by the response to disasters. Therefore, the true lesson of the TeGenero incident is that it is essential that the justification of the first-in-human dose for biotherapeutics (and for small molecules) involves the perspective of preclinical safety assessment experts experienced in the design, implementation and interpretation of the preclinical programmes for such therapies.

Christopher Horvath is at INTICA Biomedical, USA.

Laura Andrews is at Genzyme, a company of Sanofi, USA.

Andreas Baumann is at Bayer Healthcare, Germany.

Lauren Black is at Charles River Laboratories, USA.

Diann Blanset and James D. Green are at BoehringerIngelheim Pharmaceuticals, Inc., USA.

Joy Cavagnaro is at Access BIO, USA.

Kenneth L. Hastings is at Sanofi SA, USA.

David L. Hutto is at Eisai, Inc., USA.

Timothy K. MacLachlan and Mark Milton are at Novartis,

Theresa Reynolds is at Genentech, USA.

Stan Roberts is at SAR Safety Assessment, USA.

Mark Rogge is at Biogen IDEC, USA.

Jennifer Sims is at Integrated Biologix $\mathrm{GmbH}$, Switzerland.

George Treacy is at Janssen R\&D, USA.

Garvin Warner is at Alnylam Pharmaceuticals, USA.

Correspondence to C.H. and J.G. e-mails: chorvath@inticabio.com; james.green@boehringer-ingelheim.com

Disclaimer: The views expressed in this article are those of the authors and do not necessarily reflect those of their employers. doi:10.1038/nri3192-c 1 Published online 31 August 2012
1. Hünig, T. The storm has cleared: lessons from the CD28 superagonist TGN1412 trial. Nature Rev. Immunol. 12, 317-318 (2012).

2. Proft, T. \& Fraser, J. D. Bacterial superantigens. Clin. Exp. Immunol. 133, 299-306 (2003).

3. Horvath, C. J. \& Milton, M. N. The TeGenero incident and the Duff Report conclusions: a series of unfortunate events or an avoidable event? Toxicol. Pathol. 37, 372-383 (2009).

4. Suntharalingam, G. et al. Cytokine storm in a phase 1 trial of the anti-CD28 monoclonal antibody TGN 1412 N. Engl. J. Med. 355, 1018-1028 (2006).

5. Medicines and Healthcare Products Regulatory Agency. Clinical trial suspension: latest findings. MHRA [online], http://www.mhra.gov.uk/NewsCentre Pressreleases/CON2023515 (2006)

6. Lühder, F. et al. Topological requirements and signaling properties of T cell-activating, anti-CD28 antibody superagonists. J. Exp. Med. 197, 955-966 (2003).

7. Tacke, M., Hanke, G., Hanke, T. \& Hünig, T.

CD28-mediated induction of proliferation in resting $\mathrm{T}$ cells in vitro and in vivo without engagement of the $T$ cell receptor: evidence for functionally distinct forms of CD28. Eur. J. Immunol. 27, 239-247 (1997).

8. Lin, C. H. \& Hünig, T. Efficient expansion of regulatory $\mathrm{T}$ cells in vitro and in vivo with a CD28 superagonist. J. Immunol. 33, 626-638 (2003).

9. Stebbings, R. et al. "Cytokine storm" in the phase I trial of monoclonal antibody TGN1412: better understanding the causes to improve preclinical testing of immunotherapeutics. J. Immunol. 179, 3325-3331 (2007).

10. Legrand, N. et al. Transient accumulation of human mature thymocytes and regulatory T cells with CD28 superagonist in "human immune system" $\operatorname{Rag} 2^{-1-} \gamma_{c}{ }^{-1-}$ mice. Blood 108, 238-245 (2006).

11. International Conference on Harmonisation of Technical Requirements for Registration of Pharmaceuticals for Human Use. ICH harmonized tripartite guideline S6: preclinical safety evaluation of biotechnology-derived pharmaceuticals $(\mathrm{ICH}$, 1997).

12. US Food and Drug Administration Center for Drug Evaluation and Research. Guidance for industry. Estimating the maximum safe starting dose in initial clinical trials for therapeutics in adult healthy volunteers (FDA, 2005).

13. Association of the British Pharmaceutical Industry/ Biolndustry Association. Guideline for preclinical and clinical testing of new medicinal products. Parts $1 \& 2$ (ABPI, 1977).

14. Sims, J. \& ABPI/BIA Early Stage Clinical Trials Task Force. Calculation of the minimum anticipated biological effect level (MABEL) and 1st dose in humans. EMA [online], http://www.ema.europa.eu/ docs/en GB/document library/Presentation/2009/11/ WC500010862.pdf (2007)

15. Lowe, P. J. et al. On the anticipation of the human dose in first-in-man trials from preclinical and prior clinical information in early drug development. Xenobiotica 37, 1331-1354 (2007).

16. Lowe, P. J., Tannenbaum, S., Wu, K., Lloyd, P. \& Sims, J. On setting the first dose in man: quantitating biotherapeutic drug-target binding through pharmacokinetic and pharmacodynamic models. Basic Clin. Pharmacol. Toxicol. 106, 195-209 (2010).

Competing interests statement

The authors declare no competing financial interests.

\section{FURTHER INFORMATION}

Citizens for Responsible Care and Research:

http://www.circare.org/foia5/tgn1412.htm

ALL LINKS ARE ACTIVE IN THE ONLINE PDF 